In recent years, significant development in the treatment of prostate cancer has taken place. One of the most documented methods of treatment in patients characterised by a high risk of progression is a combination of radiotherapy (RT) with long-term hormone therapy (HT). In this group of patients, neither RT alone nor HT alone allows satisfactory outcomes to be achieved, and therefore as monotherapy they are not recommended as optimal methods of treatment. In this review, we summarise arguments for combining radiotherapy with hormonal therapy in high-risk prostate cancer, with an emphasis on the results of phase III trials.

Key words: prostate cancer, combination therapy, hormone therapy, radiotherapy.

Contemp Oncol (Pozn) 2015; 19 (1): 1-5 DOI: $10.5114 /$ wo.2015.50010

\section{Hormone therapy in combination with radiotherapy in the treatment of prostate cancer: why and in which group of patients?}

\author{
Tomasz Milecki ${ }^{1}$, Andrzej Antczak ${ }^{1}$, Zbigniew Kwias ${ }^{1}$, Piotr Milecki' ${ }^{2,3}$
}

${ }^{1}$ Department and Clinic of Urology and Urologic Oncology, Poznan University of Medical Science, Poznan, Poland

${ }^{2}$ Chair and Department of Electroradiology, Poznan University of Medical Sciences, Poznan, Poland

${ }^{3}$ Department of Radiotherapy, Greater Poland Cancer Centre, Poznan, Poland

In the last decades, prostate cancer (PC) has become one of the most common cancers in Europe and in the United States [1]. At the same time, hormone therapy $(\mathrm{HT})$, alongside surgery and external beam radiotherapy (RT), is the most common method used for PC therapy [2]. It is to be stressed that patients with unfavourable prognostic factors characterising PC as cancer with an intermediate or high risk of progression are predominantly treated with $\mathrm{HT}$ combined with $\mathrm{RT}[3,4]$. According to the data from the CaPSURE database, the rate of using neoadjuvant $\mathrm{HT}$ in combination with RT increased from $9.8 \%$ in $1989-1992$ to $74.6 \%$ in 1999-2001, and in the group of a high risk of progression from $15.3 \%$ in 1989-1999 to $89.5 \%$ in 1999-2001 [5]. The report by Shanini also indicates that in recent years, a continuous increase in use of HT in patients with those stages of PC was observed [6]. These data confirm the significance of HT in the treatment of PC.

Prostate cancer cells are androgen-dependent. This was already discovered in 1941 by Huggins and Hodges [7]. Having penetrated into the neoplastic cell, testosterone is converted to the more potent form known as dihydrotestosterone. Both compounds show affinity for the androgen receptor located in the cytoplasm. Together, they build a complex which after being translocated into the nucleus binds to specific DNA sequences responsible for growth, proliferation and metabolism of cancer cells. The aim of hormonal therapy for PC is to obtain the lowest possible androgen concentration in the blood via suppression of the hypothalamic-pituitary-gonadal axis (LHRH analogues) and/or inhibition of androgen receptors by testosterone-competitive and dihydrotestosterone-competitive agents (antiandrogens).

The idea of combining HT with RT was based on earlier experience with neoadjuvant HR used prior to radical prostatectomy [8]. Combination of surgery and neoadjuvant $\mathrm{HT}$, however, did not translate into significant survival improvements; therefore, neoadjuvant HT as a treatment option was abandoned. However, a positive effect of HT on pathological parameters was observed such as reduction in prostate volume including both cancerous and non-cancerous tissue, as well as a decrease in the rate of positive surgical margins [9].

One argument for neoadjuvant HT combined with RT stems from studies that showed a decrease in prostate volume, which leads to reduction in the volume of healthy tissues exposed to radiation.

The first attempts to combine HT with RT were made using animal models. Results of the study by Zietman proved that combination therapy provided a significant improvement in the efficacy of RT [10]. The study was carried out in vivo using the Shinogi murine model (with implanted prostate adenocarcinoma) which was subjected to surgical castration, and then to 
RT. Results for combination therapy were compared to the control group of patients who were subjected only to RT. The study proved that HT allows for a significant reduction of RT dose needed to destroy $50 \%$ of the tumour volume (TCD50 - the dose required for 50\% tumour control), while the greatest efficacy was observed when HT was used prior to a series of exposures to radiation (neoadjuvant therapy). The summary states that a significant role is played by the point in time when HT is initiated (neoadjuvant therapy gave better results when compared to adjuvant therapy or concomitant RT). In another study using a Dunning rat PC model, Kaminski et al. compared the efficacy of RT in combination with the following HT regimens: 1) HT used 14 days prior to RT; 2) HT during RT; 3) HT used 14 days after RT [11]. The most beneficial therapeutic effect was achieved in the case of the first method (RT + neoadjuvant $\mathrm{HT}$ ), which confirmed the results of the previous study.

One of the significant mechanisms responsible for improvement in the efficacy of combination treatment is increased oxygenation of PC cells. Hypoxia stimulates cancer cells to produce vascular endothelial growth factor (VEGF), which is responsible for tumour vascular angiogenesis [12]. Hormone therapy may have a suppressive effect on VEGF, thus reducing the process of pathological neovascularisation. As a result, HT improves cellular oxygenation, which, in turn, increases the sensitivity of neoplastic cells to RT [13]. The effect of HT on reduction of tumour volume (reduction in the number of cancer cells) is also significant and improves oxygenation of the cells that remain in the tumour [14]. This also helps to reduce the volume of healthy tissues exposed to radiation, thus reducing the adverse effects of RT [15].

Another effect of HT is its ability to induce apoptosis in cancer cells [16]. Due to this, HT eliminates cell clones which could possibly be resistant to RT. Currently, it is not known whether this is the effect of HT alone or it may be a synergistic effect of combination with RT.

Hormone therapy may also play a potential role as systemic treatment leading to apoptosis of cells producing micrometastases that cannot be targeted with RT. One possible effect of HT is as a "trigger" factor to stimulate the immunological system to fight cancer [17].

A significant prognostic parameter which may have an effect on treatment results in patients with PC when using RT is the nadir prostate-specific antigen (PSA) achieved as a result of neoadjuvant $\mathrm{HT}$. The recently published retrospective study by Zefelsky analysed this problem [18]. The study involved 1,045 patients with localised PC who were treated with neoadjuvant HT (3 months) and subsequently with RT with concomitant HT (2-3 months). Patients who achieved a nadir PSA level of $0.3 \mathrm{ng} / \mathrm{ml}$ prior to RT showed improved results. The 10-year risk of death due to RS was lower in the group of patients who achieved nadir PSA values of $\leq 0.3 \mathrm{ng} / \mathrm{ml}: 7.8 \%$ vs $13.7 \%$ in patients with nadir PSA levels $>0.3 \mathrm{ng} / \mathrm{ml}$. Similarly, the 10-year risk of biochemical recurrence in patients with PSA levels $\leq 0.3 \mathrm{ng} / \mathrm{ml}$ prior to RT was $74.3 \%$ vs. $57.7 \%(p<0.001)$ for those with levels $>0.3 \mathrm{ng} / \mathrm{ml}$, whereas the 10 -year risk of distant metastases was $86.1 \%$ vs. $78.6 \%$ in respective groups $(p<0.004)$. The results confirm that low values of PSA prior to RT and after completion of neoadjuvant $\mathrm{HT}$ have a beneficial effect on the risk of death due to $\mathrm{PC}$, reduction in distant metastases and possible biochemical recurrence.

Reductions in testosterone levels with HT may have an effect on treatment outcomes [19]. This hypothesis is being tested in metastatic PC, where the current target blood testosterone level $<50 \mathrm{ng} / \mathrm{dl}$ is considered to be too high and the adoption of a new, lower cut-off point of $20 \mathrm{ng} / \mathrm{dl}$ is proposed. The retrospective analysis evaluating the efficacy of the LHRH analogue triptorelin administered every 3 months to patients with metastatic PC showed that it led to a lower castration testosterone level $(<20 \mathrm{ng} / \mathrm{dl})$ in $95 \%$ of patients after 6 months of therapy [20].

\section{Intermediate/high-risk patient group}

In this group of PC patients, treatment outcomes with RT monotherapy are satisfactory, but further improvements in efficacy could be achieved. One possible way is by using combination therapy: HT + RT. In the DFCl 95096 study (Dana-Farber Cancer Institute), patients were randomly assigned to two study arms: RT 70 Gy alone or RT 70 Gy combined with 6-month HT (2 months of neoadjuvant HT +2 months of concomitant HT +2 months of adjuvant $\mathrm{HT}$ ) [21]. The study involved patients with PC in stage T1b2 bNOMO and with at least one unfavourable prognostic factor (PSA $10 \mathrm{ng} / \mathrm{ml}$ max. $40 \mathrm{ng} / \mathrm{ml}$, Gleason score > 7 or seminal vesicle infiltration or extracapsular infiltration confirmed in an MRI examination). After an 8-year follow-up, a significant improvement in the overall survival was observed in the group treated with combination therapy $(\mathrm{RT}+\mathrm{HT})$ when compared to the group treated with RT alone (74\% vs. 61\%; log rank $p=0.01)$. In the reanalysis of the study, the impact of concomitant diseases on treatment results was also taken into account (ACE-27 index). It was found that combination therapy improves the survival rate both in the group with an intermediate risk of progression and in the group with a high risk of progression without concomitant diseases or with minimal concomitant additional medical conditions. However, in patients affected by numerous additional medical conditions, HT may not lead to any significant improvement in the survival of these patients [22].

In another important study, TROG 9601 (Trans Tasman Radiation Oncology Group), RT alone was compared to RT combined with 3 months of HT (initiated 2 months before RT) and to RT combined with 6 months of HT (initiated 5 months before RT) [23]. The study covered PC patients with stage T2b-T4 NOMO, of whom about $20 \%$ had an intermediate risk of progression. After a 10-year follow-up period, a significant reduction in the risk of biochemical progression and in the risk of local progression was observed in the group treated with combination therapy when compared to the group treated with RT alone [24]. Results were better after 6 months of HT in comparison to 3 months of HT. Furthermore, it was observed that the 6-month HT significantly reduced the risk of distant metastases (HR 0.49), mortality due to neoplastic disease (HR 0.49) and overall mortality (HR 0.63). These improvements were not achieved in the study group treated with the 3-month HT. 
Another clinical study that deserves attention is RTOG 9408 (Radiation Therapy Oncology Group), evaluating the efficacy of 2 months of neoadjuvant HT and its further continuation for 2 months during RT [25]. Fifty four percent of patients participated in the study had an intermediate risk of progression, $35 \%$ had a low risk of progression, and $11 \%$ had a high risk of progression. The 10 -year overall survival for all groups of patients who were treated with the 4-month HT prior to RT was $62 \%$ when compared to $57 \%$ ( $p=0.03$ ) in the case of RT alone. Neoadjuvant HT also reduced mortality due to PC from $8 \%$ to $4 \%(p=0.001)$. The greatest benefit from combination therapy was achieved in the group of patients with an intermediate risk of progression (overall survival after 8 years: $72 \%$ vs. $66 \%$; $p=0.03$ ) and in the group with a high risk of progression (66\% vs. $58 \% ; p=0.03$ ). The improvement, however, was not observed in the group with a low risk of progression. Treatment-related complications were comparable in both study arms.

During the meeting of the American Society for Radiation Oncology (ASTRO) in 2013, results of the RTOG 9910 study which refer to the duration of neoadjuvant HT in patients with PC and an intermediate risk of progression were presented [26]. The randomised phase III study compared the efficacy of 8 weeks of neoadjuvant HT + RT with the present treatment regimen (28 weeks of neoadjuvant $\mathrm{HT}+\mathrm{RT}$ ). One thousand five hundered and eighty patients participated in the study, which lasted 10 years. Treatment results in both study arms were almost identical: 10-year overall survival $66 \%$ ( 8 weeks of HT) vs. $67 \%$ (28 weeks of $\mathrm{HT})(\mathrm{HR}, 0.95 ; p=0.62)$, survival free from biochemical recurrence $24 \%$ vs. $23 \%$ (HR, 0.96; $p=0.47$ ), locoregional recurrence $6 \%$ vs. $4 \%(p=0.07)$. This study challenges the meaning of neoadjuvant HT lasting more than 28 weeks in the group of patients with an intermediate risk of progression.

\section{High-risk patient group}

In this group of patients, treatment outcomes remain poor and improvements are urgently needed. Recently, a few studies were carried out that unquestionably confirmed the efficacy of combination treatment $(\mathrm{HT}+\mathrm{RT})$ in the patient group with a high risk of progression. One of the first of these studies was RTOG 8531, which involved patients with stage CT3 or stage PT3 disease with possible metastases to the lymphatic system ( $\mathrm{N}+/-)$ [23]. Hormonr therapy was initiated in the last week of RT, and was subsequently continued up to progression or until HT could no longer be tolerated - without initial determination of its minimal or maximal duration. The recently published updated results of this study also deserve attention and refer to its long-term follow-up (10 years) [27]. They indicate benefits in the group of patients treated with combination therapy when compared to the group treated only with radiotherapy with regard to the overall survival (49\% vs. 39\%) ( $p=0.002)$ and the specific survival for PC (16\% vs. $22 \%$; $p=0.0052$ ).

Another study which analysed the aspect of HT combined with RT in the group of patients with a high risk of progression was RTOG 8610 performed by Roach et al. [28]. Four hundred and seventy-one patients with PC (T2-T4) and possible metastases to the regional lymph nodes were assigned to two study arms: 1) 2 months of neoadjuvant $\mathrm{HT}+2$ months of HT during RT or 2) RT alone. The regimen of radiotherapy was performed in the same way as in the case of the RTOG 8531 study. In the group of patients treated with combination therapy, improvement in locoregional control was demonstrated after a period of 5 years ( $25 \% \mathrm{vs}$. $36 \%$ in the arm treated with RT alone) and after a period of 8 years ( $37 \%$ vs. $49 \%$ in RT alone). The 10 -year overall survival did not differ significantly ( $42.6 \%$ vs. $33.8 \%$; $p=0.12$ ); however, it was observed that in the group of patients with a Gleason score of 2-6, combination therapy significantly improved the overall survival (70\% vs. $52 \% ; p=0.01)$. In patients with a Gleason score of $7-10$, no improvement in overall survival or in locoregional control was observed.

The EORTC Group (European Organisation for Research and Treatment of Cancer) carried out two very important studies which compared the efficacy of HT in combination with RT to RT alone. The first study, EORTC 22863, involved mostly patients with a high risk of PC progression, who constituted $91 \%$ of all participants, and the remaining $9 \%$ of patients had an intermediate risk of progression [29]. A total of 415 patients were assigned to two treatment regimens: 1) RT or 2) RT combined with HT initiated on the day of radiotherapy and lasting 36 months. The results showed a significant benefit in the group treated with $\mathrm{HT}+\mathrm{RT}$ with regard to the overall survival after 5 years, which was $78 \%$ vs. $62 \%(p=0.001)$, while the overall survival after 10 years was $58 \%$ vs. $40 \%(p=0.001)$. In the second study, EORTC 22961, evaluated a 6-month HT regimen with a 3-year HT regimen [30]. All patients were initially treated with 6-months of HT initiated on the day of RT. Patients were randomised to two arms: 1) follow-up (continued up to progression of disease) or 2) a further 2.5 years of HT. Radiotherapy was performed in the same way as in the EORTC 22863 study. Hormone therapy used for 36 months showed an improvement in results when compared to 6-month HT (5-year overall survival 85\% vs. $81 \%$, respectively). The results of both studies are a solid base for the current standard of care for patients with a high risk of progression, which recommends at least 3 years of HT combined with RT. Recently, results of the newest study, PCS IV, were published, which may contribute to the verification of using such a long-term HT regimen [31]. The PCS IV study involved 630 patients with a high risk of PC progression (T3-4NO, PSA > $20 \mathrm{ng} / \mathrm{ml}$ or Gleason score > 7), in whom 18 months or 36 months of HT combined with RT was used. The study did not show statistically significant differences in the overall survival after 5 years of follow-up ( $92.1 \%$ vs. $86.8 \% ; p=0.052)$ or after 10 years of follow-up (63.6\% vs. $63.2 \% ; p=0.429)$. The authors of the study concluded that perhaps in the case of confirmation of these results, a decrease in the duration of HT might be possible. However, it should be stressed that clinical studies performed to date which are the basis for determination of the current standards of treatment indicate that long-term $\mathrm{HT}$ is still an unquestionable method of treatment when combined with RT. 
Definition of the role of RT in patients with a high risk of progression requires a separate discussion. Despite a large number of recommendations related to PC treatment in patients with a high risk of progression indicating the superiority of combination therapy (RT + HT), HT as monotherapy has been used in a substantial number of patients $[32,33]$. This is due to the lack of convincing results from clinical studies demonstrating that HT as monotherapy is less effective than combination therapy. The SPCG-7/SFUO randomised study carried out by Widmark and his team was one of the crucial trials analysing this problem. The authors compared combination therapy (RT + HT) with RT alone in a group of 977 patients (T1b - T3, N0). The study showed improvement in the overall survival and in the specific survival for PC patients treated with combination therapy $[34,35]$. Another clinical study comparing the efficacy of HT to HT alone was carried out by Mottet in a group of 264 patients (CT3-4NOMO or PT3NOMO) and showed that the 5-year survival of patients without biochemical progression according to the ASTRO definition was $60.9 \%$ in the case of combination therapy when compared to $8.5 \%$ when only HT was used ( $p<0.0001$ ) [36]. According to the Phoenix definition, the survival was $64.7 \%$ and $15.4 \%$ ( $p \leq 0.0011$ ), respectively. Improvement in the local efficacy (local failure rate $9.8 \%$ vs. $29.2 \%, p<0.0001$ ) and in the survival without distant metastases ( $3.0 \%$ vs. $10.8 \%, p<0.018$ ) was observed.

\section{Summary}

After the androgen-dependent nature of PC was discovered by Huggins and Hodges, HT became one of the crucial therapeutic interventions. The above-mentioned randomised phase III studies unequivocally confirm the superiority of combination therapy $(H T+R T)$ over RT alone. Combination of these two approaches allows for the achievement of satisfactory results in patients with an intermediate and a high risk of progression. However, the significance of the true role of HT with escalating radiation dose, especially in patients with an intermediate risk of progression, is still unknown. Results of the most recent studies allow for the assumption that a decrease in the duration of HT without a reduction in treatment efficacy may be possible.

Hormone therapy with radiotherapy combination therapy is the treatment of choice for PC patients with a high risk of progression, as both RT alone and HT alone remain suboptimal. Current guidelines define the optimal duration of HT in combination with RT in these patients as up to 36 months (3-4 months of neoadjuvant HT and its further continuation during RT and after completion of RT up to a period of 36 months) [37]. In the group of patients with an intermediate risk of progression, combination therapy is also preferred, and HT should last from 4 to 6 months (2-3 months prior to RT and its further continuation during RT).

The paper was supported by a scientific grant from IPSEN Poland.

\section{References}

1. Siegel R, Ma J, Zou Z, Jemal A. Cancer statistics, 2014. CA Cancer J Clin 2014; 64: 9-29.

2. Sharifi N, Gulley JL, Dahut WL. Androgen deprivation therapy for prostate cancer. JAMA 2005; 294: 238-44.

3. Milecki P, Kwias Z. Hormonal therapy combined with radiotherapy in locally advanced prostate cancer. Rep Pract Oncol Radiother 2002; 7: 157-63.

4. Shelley MD, Kumar S, Coles B, Wilt T, Staffurth J, Mason MD. Adjuvant hormone therapy for localised and locally advanced prostate carcinoma: a systematic review and meta-analysis of randomised trials. Cancer TreatRev 2009; 35: 540-6.

5. Cooperberg MR, Grossfeld GD, Lubeck DP, Carroll PR. National practice patterns and time trends in androgen ablation for localized prostate cancer. J Natl Cancer Inst 2003; 95: 981-9.

6. Shahinian VB, Kuo Y, Freeman JL, Orihuela E, Goodwin JS. Increasing use of gonadotropin-releasing hormone agonists for the treatment of localized prostate carcinoma. Cancer 2005; 103: 1615-24.

7. Huggins C, Hodges C. Studies on prostatic cancer: the effect of castration, of estrogen, and androgen injection on serum phosphatases in metastatic carcinoma of the prostate. Cancer Res 1941; 1: 293-7.

8. Soloway M, Sharifi R, Wajsman Z, McLeod D, Wood DP Jr, PurasBaez A. Randomized prospective study comparing radical prostatectomy alone versus radical prostatectomy preceded by androgen blockade in clinical stage B2 (T2bNxM0) prostate cancer. J Urol 1995; 154: 424-8.

9. Aus G, Abrahamsson PA, Ahlren G, Hugosson J, Lundberg S, Schain M, Schelin S, Pedersen K. Hormonal treatment before radical prostatectomy: a 3-year follow-up. J Urol 1998; 159: 2016-7.

10. Zietman AL, Prince EA, Nakfoor BM, Park JJ. Androgen deprivation and radiation therapy: sequencing studies using the Shionogi in vivo tumor system. Int J Radiat Oncol Biol Phys 1997; 38: 1067-70.

11. Kaminski JM, Hanlon AL, Joon DL, Meistrich M, Hachem P, Pollack A. Effect of sequencing of androgen deprivation and radiotherapy on prostate cancer growth. Int J Radiat Oncol Biol Phys 2003; 57: 24-8.

12. Stewart RJ, Panigrahy D, Flynn E, Folkman J. Vascular endothelial growth factor expression and tumor angiogenesis are regulated by androgens in hormone responsive human prostatę carcinoma: evidence for androgen dependent destabilization of vascular endothelial growth factor transcripts. J Urol 2001; 165: 688-93.

13. Milosevic M, Chung P, Parker C, et al. Androgen withdrawal in patients reduces prostate cancer hypoxia: implications for disease progression and radiation response. Cancer Res 2007; 67: 6022-5.

14. Sanguineti G, Marcenaro M, Franzone P, Foppiano F, Vitale V. Neoadjuvant androgen deprivation and prostate gland shrinkage during conformal radiotherapy. Radiother Oncol 2003; 66: 151-7.

15. Zelefsky MJ, Harrison A. Neoadjuvant androgen ablation prior to radiotherapy for prostate cancer: reducing the potential morbidity of therapy. Urology 1997; 49 Suppl 3 A: 38-45.

16. Isaacs JT, Lundmo PI, Berges R, Martikainen P, Kyprianou N, English HF. Androgen regulation of programmed death of normal and malignant prostatic cells. J Androl 1992; 13: 457-64.

17. Roden AC, Moser MT, Tri SD, et al. Augmentation of T cell levels and responses induced by androgen deprivation. J Immunol 2004; 173: 6098-108.

18. Zelefsky MJ, Gomez DR, Polkinghorn WR, Pei X, Kollmeier M. Biochemical response to androgen deprivation therapy before external beam radiation therapy predicts long-term prostate cancer survival outcomes. Int J Radiat Oncol Biol Phys 2013; 86: 529-33.

19. Perachino M, Cavalli V, Bravi F. Testosterone levels in patients with metastatic prostate cancer treated with luteinizing hormone-releasing hormone therapy: prognostic significance? BJU Int 2010; 105: 648-51.

20. Mounedji N, Lundstroem EA, Purcea P, Grosgurin P, Porchet HC. Efficacy of triptorelin in lowering serum testosterone (ST) in patients with advanced prostate cancer. J Clin Oncol 2011; 29 suppl 7; abstr 162.

21. D’Amico AV, Manola J, Loffredo M, Renshaw AA, DellaCroce A, Kantoff PW. 6-month androgen suppression plus radiation therapy vs radiation therapy alone for patients with clinically localized prostate cancer: a randomized controlled trial. JAMA 2004; 292: 821-7.

22. D’Amico AV, Chen MH, Renshaw AA, Loffredo M, Kantoff PW. Androgen suppression and radiation vs radiation alone for prostate cancer: a randomized trial. JAMA 2008; 299: 289-95. 
23. Denham JW, Steigler A, Lamb DS, et al. Short-term androgen deprivation and radiotherapy for locally advanced prostate cancer: results from the Trans-Tasman Radiation Oncology Group 96.01 randomised controlled trial. Lancet Oncol 2005; 6: 841-50.

24. Denham JW, Steigler A, Lamb DS, et al. Short-term neoadjuvant androgen deprivation and radiotherapy for locally advanced prostate cancer: 10-year data from the TROG 96.01 randomised trial. Lancet Oncol 2011; 12: 451-9.

25. Jones CU, Hunt D, McGowan DG, et al. Radiotherapy and shortterm androgen deprivation for localized prostate cancer. N Engl J Med 2011; 365: 107-18.

26. Pisansky TM, Hunt D, Gomella LG, et al. Radiation therapy oncology group 9910: Phase III trial to evaluate the duration of neoadjuvant total androgen suppression and radiation therapy in intermediate-risk prostate cancer. Int J Radiat Oncol Biol Phys 2013; 87: S1

27. Pilepich MV, Caplan R, Byhardt RW, et al. Phase III trial of androgen suppression using goserelin in unfavorable-prognosis carcinoma of the prostate treated with definitive radiotherapy: report of Radiation Therapy Oncology Group Protocol 85-31. J Clin Oncol 1997; 15: 1013-21.

24. Pilepich MV, Winter K, Lawton CA, et al. Androgen suppression adjuvant to definitive radiotherapy in prostate carcinoma - longterm results of phase III RTOG 85-31. Int J Radiat Oncol Biol Phys 2005; 1: 1285-90.

28. Roach M, Bae K, Speight J, et al. Short-term neoadjuvant androgen deprivation therapy and external-beam radiotherapy for locally advanced prostate cancer: long-term results of RTOG 8610. J Clin Oncol 2008; 26: 585-91.

29. Bolla M, Van Tienhoven G, Warde P, et al. External irradiation with or without long-term androgen suppression for prostate cancer with high metastatic risk: 10-year results of an EORTC randomised study. Lancet Oncol 2010; 11: 1066-73.

30. Bolla M, de Reijke TM, Tienhoven GV, et al. Duration of androgen suppression in the treatment of prostate cancer. N Engl J Med 2009; 360: 2516-27.

31. Nabid A, Carrier N, Martin AG, et al. High-risk prostate cancer treated with pelvic radiotherapy and 36 versus 18 months of androgen blockade: Results of a phase III randomized study. I Clin Oncol 2013; suppl 6; abstr 3.

32. Akaza H, Usami H, Hinotsu S, et al. Characteristics of patients with prostate cancer who have initially been treated by hormone therapy in Japan: J- CaP surveillance. Jpn J Clin Oncol 2004; 34: 329-36.

33. Klotz L, Schellhammer P, Carroll K. A re-assessment of the role of combined androgen blockade for advanced prostate cancer. BJU Int 2004; 93: 1177-82.

34. Widmark A, Klepp O, Solberg A, et al. Endocrine treatment, with or without radiotherapy, in locally advanced prostate cancer (SPCG7/SFUO-3): an open randomised phase III trial. Lancet 2009; 373: 301-8.

35. Cooperberg MR, Broering JM, Carroll PR. Time trends and local variation in primary treatment of localized prostate cancer. J Clin Oncol 2010; 28: 1117-23.

36. Mottet N, Peneau M, Mazeron JJ, Molinie V, Richaud P. Addition of radiotherapy to long-term androgen deprivation in locally advanced prostate cancer: an open randomised phase 3 trial. Eur Urol 2012; 62: 213-19.

37. National Comprehensive Cancer Network (NCCN). Practice Guidelines in Oncology: Prostate Cancer. Version 2.2014. Available at: www.nccn.org/professionals/physician_gls/pdf/prostate.pdfonOctober 17, 2014.

\section{Address for correspondence}

\section{Tomasz Milecki}

Department and Clinic of Urology and Urologic Oncology

Poznan University of Medical Sciences

Szwajcarska 3

61-285 Poznan, Poland

tel. +48 601316763

e-mail: tmilecki@wp.pl

Submitted: 15.04 .2014

Accepted: 19.11 .2014 